\title{
Results from the Final Runs of the CDMSII Experiment
}

\author{
Marek Kos ${ }^{* \dagger}$ \\ Syracuse University/CDMS \\ E-mail: mskos@phy.syr.edu
}

Results from the final runs of the CDMSII data taken at the Soudan mine were presented. The livetime for this data was $612 \mathrm{~kg}$-days. Two events were observed in the region of interest. Based on our background estimate there is a $23 \%$ probability to observe two or more background events in our signal region. We cannot therefore claim the detection of a Weakly Interacting Massive Particle (WIMP). The analysis of this data has led to a world leading limit on the spin-independent WIMP nucleon cross-section of $3.8 \times 10^{-44} \mathrm{~cm}^{2}$ at a WIMP mass of $70 \mathrm{GeV} / \mathrm{c}^{2}$.

35th International Conference of High Energy Physics

July 22-28, 2010

Paris, France

*Speaker.

$\dagger$ On behalf of the CDMS collaboration 


\section{Introduction}

Cosmological observations [1] have led to a model of the universe where about $85 \%$ of the matter is non-baryonic, non-luminous, and non-relativistic at the time of structure formation. This type of matter is generally classified as dark matter. WIMPs [2] are a candidate for dark matter that is well motivated by extensions to the Standard Model of particle physics and by thermal production models for dark matter in the early universe. The Cryogenic Dark Matter Search (CDMS) is a direct detection dark matter experiment located at a depth of 2090 meters water equivalent (mwe) at the Soudan mine in Minnesota, USA.

\section{The CDMS Detectors}

The CDMSII detectors consist of $19 \mathrm{Ge}$ detectors of about $230 \mathrm{~g}$ each and $11 \mathrm{Si}$ of about $100 \mathrm{~g}$ each. For this analysis data was used from a subset of the Ge detectors that met our data quality requirements. The CDMSII detectors are cylinders $1 \mathrm{~cm}$ thick and 3 inches $(7.62 \mathrm{~cm})$ in diameter. The top of these detectors are instrumented with phonon detectors readout in 4 phonon channels in a quadrant design. To measure the ionization signal an electric field is applied to these detectors via 2 concentric electrodes at the bottom.

\section{Background Rejection}

To be sensitive to possible WIMP interactions electron-recoil background events are rejected from nuclear-recoil events (WIMP type events). This rejection is done by reading out the energy deposited in the charge and phonon channels separately. Nuclear-recoil events deposit most of their energy as phonons, with reduced charge energy. Bulk electron recoil events have a higher charge signal. The ratio of charge to recoil energy, ionization yield, provides better than 10000 to 1 rejection of electron recoil events.

Electron-recoil events within a few $\mu \mathrm{m}$ of the detector surface can have incomplete charge collection. Therefore these surface events can have reduced ionization yield and be misclassified as nuclear-recoil events. Fortunately, since these events occur near the surface, they also have faster phonon rise times than bulk events. The surface events can therefore be rejected based on phonon timing parameters. The rejection based on phonon timing is about 200 to 1 . Figure 1 shows the rejection based on ionization yield and timing.

\section{Analysis of Data in Signal Region}

The WIMP-search data selection cuts were developed prior to unblinding of the WIMP region of interest. Candidate events had to pass data quality and fiducial volume cuts, the energy of the event can only be deposited in one detector (single scatter), the event must not be coincident with a hit in a veto panel, ionization yield must be within $\pm 2 \sigma$ of the mean yield determined from neutron calibration data, and finally the event has to pass the phonon timing cut. After unblinding 2 events were observed in the signal region. One event was in the fifth detector in tower 1 (T1Z5), and the other was in the fourth detector in tower 3 (T3Z4). Figure 2 shows the data after unblinding in ionization yield and phonon timing. 

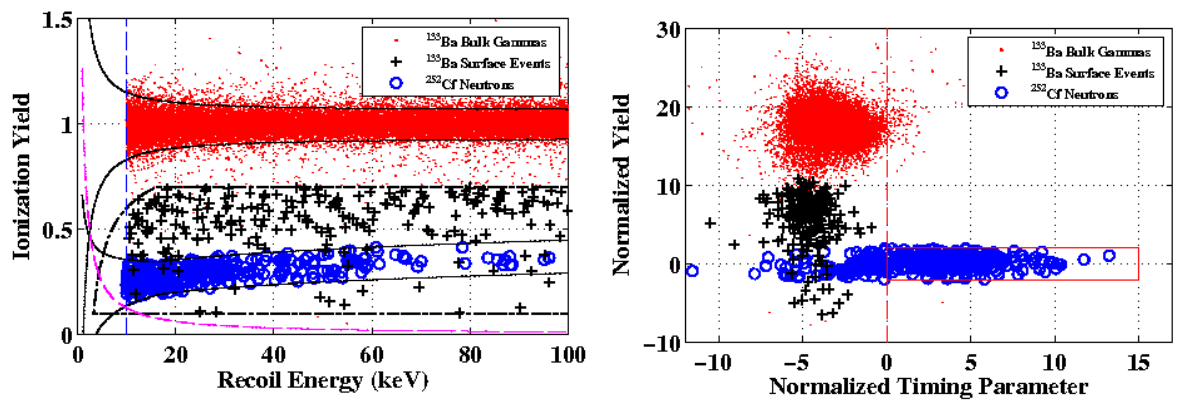

Figure 1: The left panel shows ionization yield as a function of recoil energy for ${ }^{133} \mathrm{Ba}$ and ${ }^{256} \mathrm{Cf}$ calibration sources. There is clear separation between the ${ }^{133} \mathrm{Ba}$ gamma events and neutrons (nuclear recoils) from ${ }^{256} \mathrm{Cf}$. Events that make it down to the nuclear recoil band are surface events. The right panel shows that these surface events can be rejected based on phonon timing.
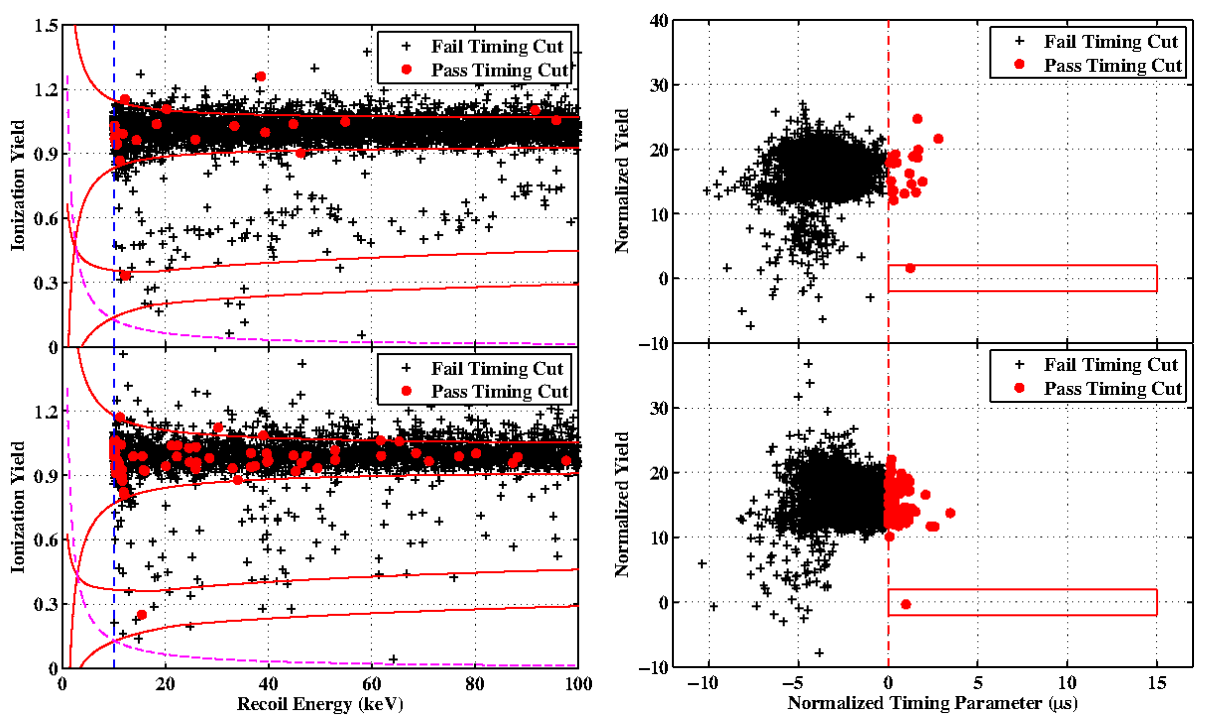

Figure 2: Left panel: ionization yield as a function of recoil energy for the unblinded data. Right panel: Yield as a function of timing parameter for the ublinded data.

The surface background estimate in the signal region is $0.8 \pm 0.1$ (stat) \pm 0.2 (sys). After including the expected neutron background, 0.04 events, the probability to observe 2 or more background (neutron and surface) events in our signal region is $23 \%$. Thus we cannot claim a WIMP detection. A likelihood analysis was done on the 2 events comparing the events to timing and yield distributions from neutron calibration data. The probability that a true nuclear recoil event in our signal region is as close to the cut boundaries as the event observed in T1Z5 is 4\%, and for the event in T3Z4 it is $19 \%$. This further suggests that the observed events are due to background.

\section{WIMP Sensitivity}

The spin-independent sensitivity including the final CDMSII data is shown in figure 3. The 

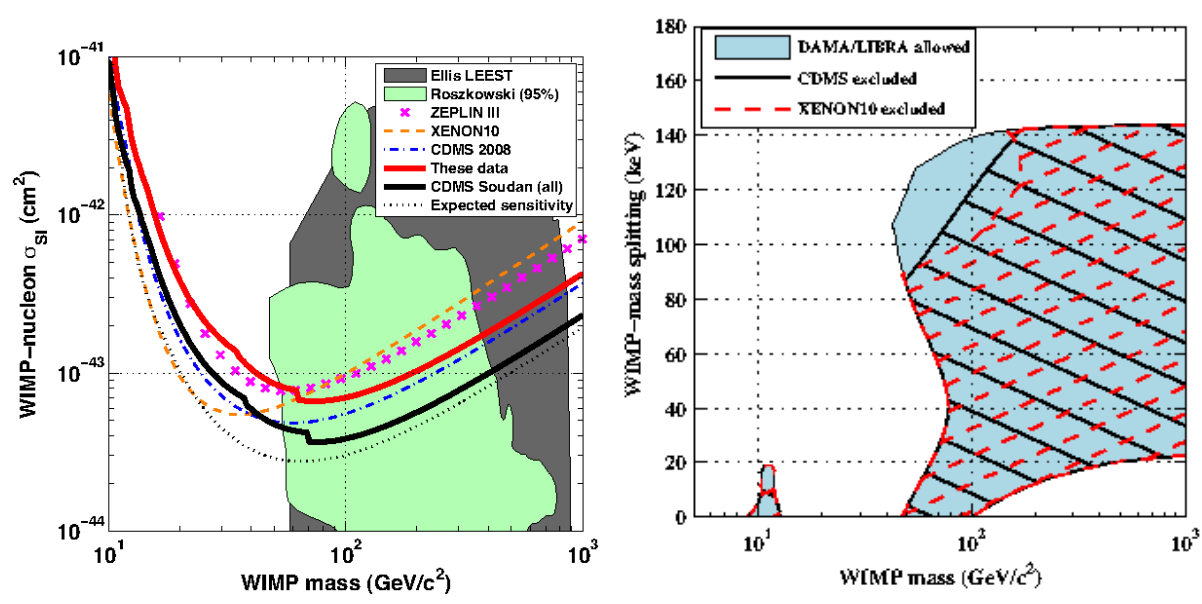

Figure 3: Left panel: 90\% C.L. upper limits on the WIMP-nucleon spin-independent cross section as a function of WIMP mass. The red line shows the limit obtained from the final CDMSII runs at Soudan (July 2007 - September 2008). The solid black line shows the limit from the combined CDMS runs at Soudan. Right panel: The blue region represents WIMP masses and mass splittings under the inelastic dark matter interpretation compatible with the DAMA modulation signal at 90\% C.L. The hatched regions are excluded by CDMSII and XENON10 [5]

upper limit on the WIMP-nucleon elastic scattering cross section is $7.0 \times 10^{-44} \mathrm{~cm}^{2}$ for a WIMP mass of $70 \mathrm{GeV} / \mathrm{c}^{2}$ at $90 \%$ C.L.

As a way to reconcile the possible DAMA detection of a WIMP signal and the failure of other experiments to observe a signal it has been proposed by Weiner et al. [4] that WIMPs scatter via a transition to an excited state with mass splitting $\delta$. Figure 3 shows the DAMA allowed region in WIMP mass and mass splitting $\delta$ and the region excluded by the CDMSII data. All but a narrow region of low mass and high mass splitting is excluded.

\section{References}

[1] E. Komatsu et al. (WMAP), Astrophys. J. Suppl. 180330 (2009)

[2] G. Steigman and M.S. Turner,Nucl. Phys. B 253375 (1985)

[3] CDMSII Collaboration, Science 3271619 (2010)

[4] S. David and N. Weiner, Phys. Rev. D. 64043502 (2001)

[5] J. Angle et al., Phys. Rev. D. 90115005 (2009) 